\title{
Immune mechanisms in tienilic acid associated hepatotoxicity
}

\author{
JAMES NEUBERGER AND ROGER WILLIAMS \\ From the Liver Unit, The Queen Elizabeth Hospital, Queen Elizabeth Medical Centre, Birmingham and the \\ Liver Unit, King's College Hospital and School of Medicine and Dentistry, London
}

SUMMARY In order to investigate the mechanisms underlying the hepatotoxicity associated with tienilic acid (Ticrynafen) ingestion we have looked for evidence of sensitisation to drug altered liver cell determinants using an indirect antibody dependent, cell mediated cytotoxicity assay (ADCC). As targets, hepatocytes were isolated from rabbits pretreated with either tienilic acid or its isomer with or without previous enzyme induction with either phenobarbitone or B-naphthoflavone (BNF). Sera from 16 of 36 patients with presumed tienilic acid hepatotoxicity induced significant cytotoxicity to hepatocytes isolated from rabbits pretreated with BNF and subsequently tienilic acid. Three of 10 sera from patients receiving tienilic acid but without overt liver damage also induced significant cytotoxicity to these hepatocytes, however, although none of 20 normal controls or of 16 patients with other liver diseases did so. Non-organ specific autoantibodies, classified as anti-LKM2, were also detectable. These were present in association with tienilic acid associated antibodies: of the 36 patients with presumed tienilic acid hepatotoxicity, $38 \%$ had both antibodies, $18 \%$ had only antiLKM 2 antibodies and $9 \%$ only tienilic acid associated antibodies. These results suggest that this drug reaction is associated with sensitisation to drug altered liver cell antigens and autoantigens. If ticrynafen associated hepatotoxicity is immune mediated, then one possible mechanism is that the drug induced antigens break tolerance, leading to an immune attack on normal liver cell components.

Tienilic acid, also known as Ticrynafen, is a uricosuric diuretic which has been widely and effectively used in the management of patients with hypertension.' Its use has been restricted by the occasional report of drug associated hepatotoxicity, however, which is characterised by fever, abdominal pain, jaundice, rise in serum transaminases and a histological picture resembling viral hepatitis. ${ }^{.-4}$ Until 1980, when the drug was withdrawn from sale in the United States, there were 340 instances of hepatic injury considered as likely to be drug related in an estimated exposed population of $265000 .^{7}$ This frequency of 1 in 800 in the USA is considerably greater than that observed in France (about one in 10000). ${ }^{\circ}$ While this difference may merely reflect a more efficient reporting system in the United States than in France, an alternative explanation is more likely to

Address for correspondence: Dr Roger Williams, Liver Unit, King's College Hospital, School of Medicine and Dentistry, Denmark Hill, London SE5 8RX. Accepted for publication 30 September 1988. be the slightly greater amount of isomer of tienilic acid present in the preparation available in the US.

Features of tienilic acid associated liver damage include the presence of fever, a prompt recurrence of clinical and serological features on challenge, eosinophils on the liver histology and the presence of nonorgan specific autoantibodies. These autoantibodies include the recently described anti-liver-kidney microsomal antibodies which have a different specificity to that associated with the LKM antibodies found in other conditions and are therefore termed 'anti-LKM2' ${ }^{121.13}$ These LKM-2 antibodies cease to be detectable within three to nine months after stopping the drug." These features have been thought by some ${ }^{12}$ to be compatible with an immune mechanism; others ${ }^{7}$ have drawn attention to the lack of peripheral eosinophilia and rash and the variable and prolonged latent period which may be more in keeping with a mechanism other than immune mediated toxicity. These latter authors did, however, 
suggest that hypersensitivity may play a role in some patients.

In some other instances of immune mediated drug hepatotoxicity, notably associated with halothane and methyldopa, it has been possible using antibodydependent cell mediated cytotoxicity to demonstrate the presence of antibodies reacting with drug altered liver cell determinants. ${ }^{1+15}$ In this report, we have looked for evidence of sensitisation to tienilic acid associated liver cell determinants in the serum of patients with such drug induced liver damage and compared the presence of these antibodies with that of anti-LKM. With few exceptions, metabolism is required for expression of drug related toxicity. Tienilic acid is metabolised by the mixed function oxidase system. ${ }^{16}{ }^{17}$ Activity of this system can be enhanced by pretreatment with different enzyme inducers. While phenobarbitone will enhance the cytochrome P-450 activity, drugs such as Bnaphthoflavone will enhance the P-448 activity, stimulating tienilic acid metabolism through different pathways, with different metabolites. To determine whether tienilic acid metabolism was important in the generation of any putative antigen, we have examined the effect of these two inducers in the generation of tienilic acid associated antigens.

\section{Methods}

\section{PATIENTS}

Sera were made available from 40 patients who had developed hepatotoxicity presumably associated with tienilic acid ingestion and in none was there evidence of other causes for the liver damage. There were 17 men and ages ranged from 32 to 84 years (median 69). The patients had been taking tienilic acid at a median dose of $250 \mathrm{mg} /$ day (range 125 to 500 $\mathrm{mg} /$ day). Abnormal liver function tests were first noted between 30 days and four years (median three months) after starting treatment. Twenty four had jaundice and the maximum rise in serum transaminase was 2013 IU/l. Twenty five of the 40 patients had detectable concentrations of circulating antiLKM2 antibodies detected by indirect immunofluorescence." One additional serum which had circulating anti-LKM2 antibodies was discarded because the patient had not been taking tienilic acid. Additional serum was available in 10 patients who had taken tienilic acid ( $250 \mathrm{mg} /$ day) for a minimum period of four months and all had normal liver function tests. Because some sera were available in limited supply, it was not possible to test all sera against all hepatocytes. As controls, serum was taken from 20 normal subjects and 16 patients with liver cell necrosis caused by toxicity from other drugs (paracetamol four, halothane three, rifampicin/ isoniazid two, ampicillin one, carbon tetrachloride one, alcohol five).

PREPARATION OF SERUM

Serum was stored until use at $-20^{\circ} \mathrm{C}$. Immediately before use the sera were heated at $56^{\circ} \mathrm{C}$ for 30 minutes to inactivate complement. In order to remove antibodies reacting with normal rabbit liver cell determinants, $200 \mu \mathrm{l}$ serum was incubated at room temperature with an equal volume of hepatocytes isolated by collagenase digestion (as outlined below) for 30 minutes, and after spinning at $1600 \times g$ for five minutes the serum was then incubated for a further 30 minutes with an equal volume of hepatocytes at $4^{\circ} \mathrm{C}$. After further centrifugations the serum was diluted 1:100 in RPMI 1640 containing glutamine and antibiotics. The absence of antibodies reacting with normal rabbit liver cells was confirmed by the inability of the serum to induce significant cytotoxicity to control rabbit hepatocytes.

TREATMENT OF ANIMALS

Female New Zealand white rabbits, weight 2 to $2 \cdot 5$ $\mathrm{kg}$, were treated with tienilic acid or its isomer (kindly provided by Anphar Rolland Laboratories, Chilly, France), either as an oral dose of $250 \mathrm{mg} / \mathrm{kg}$ / day for seven days or by a single intraperitoneal injection of tienilic acid or its isomer $(100 \mathrm{mg} / \mathrm{kg})$, either after no pretreatment, pretreatment with Bnaphthoflavone $80 \mathrm{mg} / \mathrm{kg}$ by intraperitoneal injection (ip) in $5 \mathrm{ml}$ corn oil 48 hours before giving the drug, or phenobarbitone (ip) $100 \mathrm{mg} / \mathrm{kg} /$ day for three days before giving tienilic acid or its isomer. Tienilic acid or isomer were suspended in $5 \mathrm{ml}$ sterile water and fed to the animals by gavage. Twelve hours after the last dose of tienilic acid (or isomer) the rabbits were killed by intravenous injection of $240 \mathrm{mg}$ pentabarbitone and $1000 \mathrm{IU}$ preservative free heparin. The liver was removed with full sterile technique and, after perfusion with RPMI 1640 medium, the liver was minced and hepatocytes isolated by four hours digestion with $0.01 \%$ collagenase type IV (Sigma Chemical Co) as described elsewhere. ${ }^{14}$ Ten millilitres isolated hepatocytes were then seeded into wells of a microcytotoxicity plate at a concentration of 200 hepatocytes per well. The purity of the hepatocytes, as judged by electron microscopy, was over $95 \%$ and the viability, as judged by trypan blue exclusion was over $90 \%$. The cells were maintained at $37^{\circ} \mathrm{C}$ in $95 \%$ oxygen, $5 \%$ carbon dioxide.

\section{CYTOTOXICITY REACTION}

After overnight incubation of the hepatocytes in the microcytoxicity plates the supernatant was removed and the hepatocytes incubated for two hours at $37^{\circ} \mathrm{C}$ with either $10 \mu$ l diluted serum or, as control, $10 \%$ 
heat inactivated fetal calf serum (Gibco, Paisley, Scotland) in RPMI 1640 in order to allow any antibody present in the serum to react with the respective antigens. The supernatant was removed, the remaining hepatocytes washed in $10 \%$ fetal calf solution and then incubated with lymphocytes, isolated from one normal individual who had never been exposed to tienilic acid and whose lymphocytes were not cytotoxic to hepatocytes. Lymphocytes were isolated by dextran sedimentation, Ficoll Triosil centrifugation and petri dish incubation, in order to allow those lymphocytes with $\mathrm{Fc}$ receptors to bind to and lyse the antibody coated target cells. After 36 hours' incubation the plates were inverted for one hour, washed and the remaining adherent hepatocytes fixed with $100 \%$ methanol and stained with $5 \%$ eosin. The hepatocytes were counted without the observer having knowledge of the source of serum. All serum samples were tested in at least 10 wells. The percentage cytotoxicity was calculated from the formula:

$$
\% \text { cytotoxicity }=\frac{\mathrm{NC}-\mathrm{NT}}{\mathrm{NC}} \times 100
$$

where $\mathrm{NC}=$ number of cells in the control wells and $\mathrm{NT}=$ number of cells in the test wells.

The upper limit of normal, determined as the mean plus two standard deviations obtained from 30 normal sera, was $28 \%$. Values of $28 \%$ and above were considered to have significant cytotoxicity. Repeated testing of 20 sera showed no change in cytotoxicity of the samples.

\section{Results}

None of the 19 sera from patients with tienilic acid hepatotoxicity induced significant cytotoxicity to hepatocytes isolated from rabbits pretreated with a single dose of tienilic acid or its isomer (\% cytotoxicity $0-18$, median 11 and $0-24$, median 9 respectively). When hepatocytes isolated from rabbits pretreated with tienilic acid for seven days were used as the target cells, three of $24(12.5 \%)$ sera induced significant cytotoxicity. When the target hepatocytes isolated from rabbits pretreated with tienilic acid isomer were used, however, significant cytotoxicity was induced in three additional sera.

When hepatocytes from rabbits pretreated with BNF and tienilic acid were used as target cells, 16 of 34 sera induced significant cytotoxicity (Figure). Thus sera from 13 of the 19 patients with tienilic acid hepatotoxicity and with detectable anti-LKM2 and three of 13 without LKM2 antibodies contained antibodies reacting with drug induced liver cell determinants. In addition, three of 10 sera from patients receiving tienilic acid but without evidence

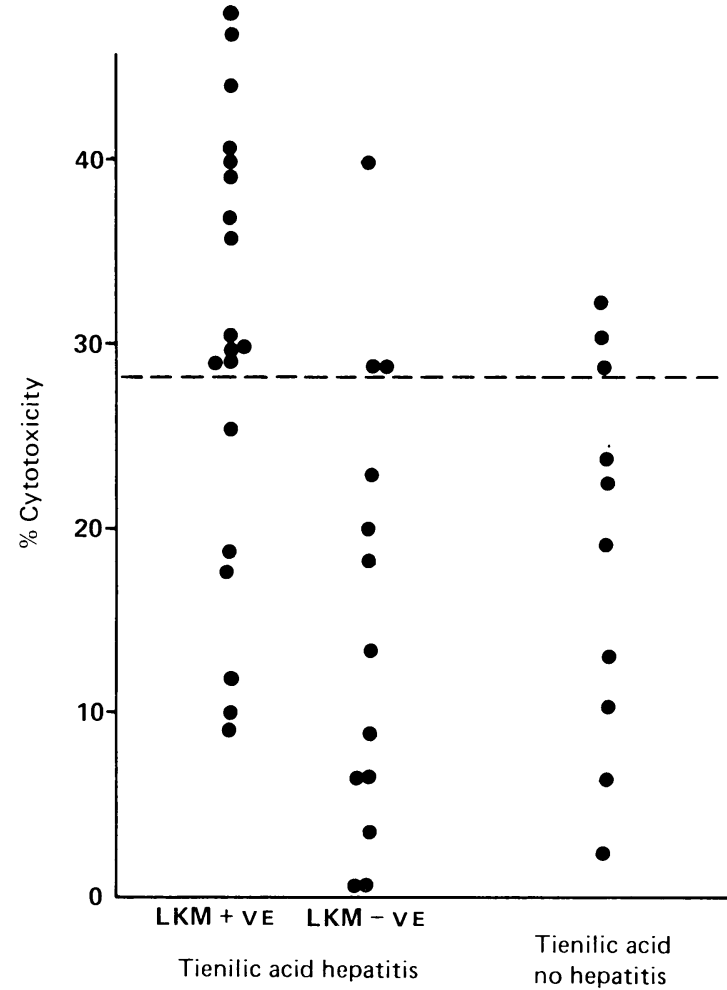

Figure Percent cytotoxicity to hepatocytes, isolated from rabbits pretreated with $B$-naphthoflavine and tienilic acid, by serum from patients with presumed tienilic acid associated hepatitis with and without the LKM-2 antibody and patients taking tienilic acid without hepatitis. ....... represents the upper limit of normal.

of liver damage contained tienilic acid associated antibodies. None of the sera induced significant cytotoxicity to hepatocytes from rabbits pretreated with BNF and tienilic acid isomer.

After phenobarbitone pretreatment none of the sera induced significant cytotoxicity to liver cells from rabbits given either tienilic acid or its isomer (\% cytotoxicity $0-24$, median 3 , and $0-23$, median 10 respectively). None of the control sera induced significant cytotoxicity to any of the target cells ( $\%$ cytotoxicity $2-24$, median 16 ).

The presence of the antibody to tienilic acid treated antigens did not correlate with the age or sex of the patient or duration of drug ingestion.

In order to show that the induced cytotoxicity was antibody dependent, five sera inducing significant cytotoxicity to hepatocytes from rabbits pretreated with tienilic acid and BNF were incubated with these hepatocytes and, after washing, the antibody coated target cells were further incubated for two hours with 
$2 \mu \mathrm{g}(\mathrm{ab})_{2}$ component of antihuman $\mathrm{Fc}$ in order to coat the Fc portion of the antibody and so prevent reaction of the killer cells with the Fc portion of the antibody. These cells were then incubated with lymphocytes and the cytotoxicity experiment performed as above. After this procedure none of the five sera induced significant cytotoxicity from 32$40 \%$ cytotoxicity to $2-16 \%$ after incubation with $\mathrm{F}(\mathrm{ab})_{2}$ anti $\mathrm{Fc}$.

\section{Discussion}

These results show that some patients with presumed tienilic acid associated hepatotoxicity have circulating antibodies reacting with drug altered liver cell determinants present on the cell surface of rabbits pretreated with tienilic acid after enzyme induction. The antibody is detectable mainly in those who also have the anti-LKM2. As the human antibody reacts with a determinant present on rabbit liver cell membrane, it is likely that a similar, if not identical antigen is present on human hepatocytes. Although the physiological relevance of the antibody dependent cell mediated cytotoxicity assay (ADCC) reaction and, in particular, the significance of the end point (loss of adhesion of isolated hepatocytes to tissue culture plastic) remain to be established, the blocking studies clearly show that the system is suitable for detecting antibody/antigen interactions of at least one subclass of antibodies. The system has been validated by use of other techniques in the investigation of antibodies to other drug altered liver cell membrane determinants using an enzyme linked immunosorbent assay and immunoblotting. ${ }^{18}{ }^{19}$ While it still remains to be established whether these antibodies do play a role in the pathogenesis of the liver damage, their ability to induce a subpopulation of normal lymphocytes to become cytotoxic to antibody coated target cells in vitro raises the possibility that these antibodies may be involved in the pathogenesis of the liver damage. Nevertheless, their presence indicates that immune recognition of drug altered liver cell antigens has occurred.

The antibody was not detectable in the serum of all cases of presumed tienilic acid hepatotoxicity. This could be because complexing of antibody on to the surface of the patient's hepatocyte, or the antibodies could be present in the circulation as circulating immune complexes. It has been assumed in all cases studied that the drug was responsible for the hepatic damage, and the possibility of other unidentified agents being implicated in this damage cannot be excluded. Furthermore, some sera were tested after the drug had been discontinued so the stimulus to antibody production may have ceased. Finally, as discussed below, tienilic acid hepatotoxicity may be mediated in some, if not all, cases by mechanisms other than immune.

Expression of the drug related antigen is dependent upon metabolism of the drug, because the number of sera with detectable concentrations of antibody is greater when using hepatocytes isolated from rabbits pretreated with the enzyme inducer B-naphthoflavone. This indicates that the antigen recognised by the antibodies in the sera is generated by metabolism of tienilic acid through a route preferentially stimulated by BNF. It has been established that the drug is metabolised through the cytochrome P-450 mixed function oxidase system, and covalent binding of reactive metabolites to liver cell macromolecules occurs. ${ }^{16}$ If, therefore, covalent binding will render these macromolecules immunogenic, either by acting as a hapten or by alteration of the quarternary structure, these macromolecules will be translocated and incorporated in the liver cell membrane through the usual biosynthetic pathways,$^{20}$ thus resulting in the appearance of an immunogen on the cell surface. Recognition of these immunogens may lead to the development of an immune mediated attack upon the cell membrane.

Although we have shown that such antibodies are present in the serum of some patients with tienilic acid associated hepatotoxicity, it cannot necessarily be assumed that these antibodies are any more than a secondary phenomenon. Indeed, the finding that not all the patients have antibodies to either normal or drug related antigens suggests that there may be two different mechanisms of toxicity. Zimmerman et al ${ }^{16}$ have shown that perfusion of the isolated rat liver with tienilic acid led to a reduction in bile flow and release of aminotransferase activity. This was enhanced by pretreatment with phenobarbitone and these findings provide an alternative explanation for the hepatotoxicity. It is possible to develop a hypothesis for a model of immune mediated liver damage in such patients.

Recent evidence suggests that, in the normal individual, all the components required for an autoimmune response are present but held in check..$^{2122}$ This restraint may be bypassed, either by a defect in the host suppressor function or by bypassing the normal regulatory mechanisms. Hence, tienilic acid ingestion may lead to the generation of a drug related antigen on the hepatocyte surface which is recognised by T lymphocytes and will, in susceptible individuals, lead to hepatocellular necrosis. This $T$ cell reaction may also stimulate $B$ lymphocytes to react against normal liver cell components such as the antigen recognised by the LKM2 antibody. Recent evidence suggests that the antigens, recognised by the antiLKM antibodies are in the endoplasmic reticulum and, indeed, may represent one form of the cyto- 
chrome P-450 enzymes. ${ }^{23}$ There is no clear evidence that cytochrome P-450 appears on the liver cell plasma membrane and so it is unlikely that this antigen represents a target for immune mediated liver disease; these antibodies may therefore develop as a consequence of tienilic acid induced hepatotoxicity.

The finding of antibodies to drug related antigens in the serum of patients without overt liver damage contrasts with the findings in patients with other forms of drug induced liver damage which may be immunologically mediated such as halothane and $\alpha$ methyldopa associated hepatitis. ${ }^{1+15}$ In the latter two conditions, it seems very likely that the cytotoxic reaction is directed against the drug-altered antigen; if tienilic acid induced hepatitis is immune mediated, then the drug associated antigen is unlikely to be the target of the immune response but may act as a trigger to break tolerance.

$\mathrm{JN}$ is a Wellcome senior clinical research fellow. We are grateful to Dr J P Labaune and Dr J Levy for providing the serum samples, and for helpful advice. We are also grateful to Professor J C Homberg, Hôpital Saint-Antoine, Paris, France, for testing the serum for the presence of anti-LKM2.

\section{References}

1 Nemati M, Kyle M, Freis E. Clinical study of ticrynafen - a new diuretic, antihypertensive and uricosuric agent. JAMA 1977; 237: 6.52-3.

2 De Carvalho S, Dunn F, Carysant S, Frohlich E. Ticrynafen - a novel uricosuric antihypertensive naturetic agent. Arch Intern Med 1978; 138: 53-6.

3 Pariente EA, Andre C. Zafrani EJ, et al. Tienilic acid can induce acute hepatitis, chronic hepatitis and cirrhosis: a report of three cases. Gastroenterol Clin Biol 1981; 5: 567-71.

4 Eugene C, Patriarche C, Bergue A, et al. Tienilic acid as a likely cause of 'Autoimmune' chronic active hepatitis. Nouv Presse Med 1980; 9: 1885-7.

5 Bousquet O, Saigot T. Bernard F, et al. Hepatitis during treatment with tienilic acid. Therapie 1980; 35: $205-8$.

6 Mechali D, Marche C, Metayer J, et al. Hepatite cytolytique au cours d'un traitement par l'acide ticlinique. Therapie 1980; 35: 197-203.

7 Zimmerman HJ, Lewis JH, Ishak KG, Maddrey W. Ticrynafen-associated injury: analysis of 340 cases. Hepatology 1984; 4: 315-23.

8 Ticrynafen Recalled. FDA Drug Bull 1980; 10: 3-4.

9 Bader JP. Les complications associees a l'emploi de l'acide tienilique. Concours Med 1982: 104-20.
10 Poupon R. Homberg JC, Abuaf N, et al. Tienilic acidinduced hepatitis associated with liver-kidney microsomal antibody: 6 cases. Nouv Presse Med 1980; 9: $1881-4$.

11 Homberg JC, Andre C, Abuaf N. A new anti-liverkidney microsome antibody (anti-LKM-2) in tienilic acid induced hepatitis. Clin Exp Immunol 1984; 55: 56170.

12 Lafay JP, Poupon R, Legendre C. Homberg JC. Darnis C. Atteintes hepatiques associces aux anticorps antimicrosomes de foic et de rein (type 2) et a la prise dacide tienilique. Gastroenterol Clin Biol 1983; 7: 523-8.

13 Smith MGM, Williams R, Walker JG, Doniach D. Hepatic disorders associated with liver/kidney microsomal antibodies. Br Med J 1974; ii: 80-3.

14 Vergani D, Mieli-Vergani G, Alberti A, et al. Antibodies to the surface of halothane altered rabbit hepatocytes in patients with severe halothane associated hepatitis. N Engl J Med 1980; 303: 66-71.

15 Neuberger J, Kenna JG, Nouri-Aria K, Williams R. Antibody mediated hepatocyte injury in $\alpha$-methyl dopa induced hepatotoxicity. Gut 1985; 26: 1233-6.

16 Zimmerman HJ, Abernathy CO, Lukacs L, Ezekiel M. Effects of ticrynafen on hepatic excretory function in the isolated perfused rat liver. Hepatology 1982; 2: $255-7$.

17 Dubb JW, Stote RM, Maass AR, Hwang BV, Familiar RG, Alexander R. Tienilic acid: pharmacokinetics, salicylate interaction and creatinine secretion studies. Postgrad Med J 1979; 55: 47-57.

18 Kenna JG, Neuberger J, Williams R. Characteristics of halothane induced antigens by immunoblotting. Biochem Soc Trans 1985; 12: 910-1.

19 Kenna JG, Neuberger J, Williams R. An enzyme-linked immunosorbent assay for detection of antibodies against halothane altered hepatocyte antigens. $J$ Immunol Meth 1984; 75: 3-14.

20) Morre DJ, Kartenbeck J, Franke WW. Membrane flow and interconversions among endomembranes. Biochem Biophys Acta 1979; 544: 71-152.

21 Allison AC, Denman AM, Barnes RD. Co-operating and controlling functions of thymus-derived lymphocytes in relation to autoimmunity. Lancet 1971; ii: 135-7.

22 McFarlane IG. Autoimmunity in liver disease. Clin Sci 1985; 67: 569-78.

23 Alvarez F, Bernard O, Homberg JC, Kreibich G. Anti liver-kidney microsome antibody recognises a 50,000 molecular weight protein of the endoplasmic reticulum. $J$ Exp Med 1985; 161: 1231-6.

24 Dausette D, Beaune P, Kiffel L, et al. Human antiendoplasmic reticulum autoantibodies appearing in hepatitis induced by tienilic acid are directed against a human liver cytochrome $\mathbf{P} 450$ hydroxylating the drug. Hepatology 1986; 3: 42. 